Elsevier Editorial System(tm) for

International Journal of Pediatric Otorhinolaryngology

Manuscript Draft

Manuscript Number: IJPORL-D-19-00803R2

Title: High frequency of paediatric facial nerve palsy due to Lyme disease in a geographically endemic region

Article Type: Full Length Article

Keywords: Facial nerve palsy, Lyme disease, Bells palsy

Corresponding Author: Dr. Alasdair Munro, B.M, B.SC

Corresponding Author's Institution: University Hospital Southampton

First Author: Alasdair Munro, B.M, B.SC

Order of Authors: Alasdair Munro, B.M, B.Sc; Robert Dorey; Daniel Owens; Duncan Steed; Christina Petridou; Trent Herdman; Christine Jones; Sanjay Patel; Kate Pryde; Saul Faust

Abstract: Introduction: Idiopathic facial nerve palsy (FNP) is an uncommon but important presentation in children, with Lyme disease known to be a common cause. The UK county of Hampshire is a high incidence area of Lyme disease. We conducted a retrospective review of the investigation and management of paediatric FNP at a large University hospital, including serologic testing and treatment of Lyme disease.

Methods: We conducted a retrospective chart review of children under 18 presenting between January 1st 2010 and December 31st 2017 with a diagnosis of FNP. Patients with clear non-Lyme aetiology at presentation were excluded. Data was collected on demographics, initial presentation, investigations including Lyme serology, and management.

Results: A total of 93 children were identified, with an even proportion of male to female and median age 9.3 years (IQR 4.6-12 years). A history of rash was present in 5.4\%, tick bite in $14 \%$ and recent travel to, or residence in the New Forest in 22.6\%. Lyme serology was performed in $81.7 \%$ of patients, of which 29\% were positive. Antibiotics were prescribed for $73.1 \%$ of patients, oral steroids for $44 \%$ and aciclovir for $17.2 \%$.

Conclusion: Lyme disease is a significant cause of FNP in this endemic area of the UK, and there was a large degree of variability in management prior to national guideline publication. Areas with endemic Lyme disease should consider introducing local guidelines supporting routine investigation and management for FNP, including empiric treatment for Lyme disease in accordance with NICE guidelines to improve care and reduce variability. 


\section{Title: High frequency of paediatric facial nerve palsy due to Lyme disease in a geographically endemic region}

Authors: Alasdair PS Munro ${ }^{1,2,3}$, Robert B Dorey ${ }^{1,2}$, Daniel R Owens ${ }^{1}$, Duncan J Steed ${ }^{3}$, Christina Petridou ${ }^{4}$, Trent Herdman ${ }^{4}$, Christine E Jones $^{2,3}$, Sanjay V Patel ${ }^{2}$, Kate Pryde ${ }^{2}$, Saul N Faust $^{1,2,3}$

Affiliations: ${ }^{1}$ NIHR Southampton Clinical Research Facility, University Hospital Southampton NHS Foundation Trust, Southampton, United Kingdom. ${ }^{2}$ Paediatric Medicine, University Hospital Southampton NHS Foundation Trust, Southampton, United Kingdom. ${ }^{3}$ Faculty of Medicine and Institute for Life Sciences, University of Southampton, Southampton, United Kingdom. ${ }^{4}$ Public Health England Rare and Imported Diseases Laboratory, Porton Down, Salisbury, United Kingdom.

Address correspondence to: Dr Alasdair Munro, NIHR Southampton Clinical Research Facility, University Hospital Southampton NHS Foundation Trust, Tremona Road, Southampton, Hampshire, UK, SO166YD [a.munro@doctors.org.uk].

Word count: 1318

Conflicts of interest: The authors have no conflicts of interest to declare 


\begin{abstract}
Introduction: Idiopathic facial nerve palsy (FNP) is an uncommon but important presentation in children, with Lyme disease known to be a common cause. The UK county of Hampshire is a high incidence area of Lyme disease. We conducted a retrospective review of the investigation and management of paediatric FNP at a large University hospital, including serologic testing and treatment of Lyme disease.
\end{abstract}

Methods: We conducted a retrospective chart review of children under 18 presenting between January $1^{\text {st }} 2010$ and December $31^{\text {st }} 2017$ with a diagnosis of FNP. Patients with clear non-Lyme aetiology at presentation were excluded. Data was collected on demographics, initial presentation, investigations including Lyme serology, and management.

Results: A total of 93 children were identified, with an even proportion of male to female and median age 9.3 years (IQR $4.6-12$ years). A history of rash was present in $5.4 \%$, tick bite in $14 \%$ and recent travel to, or residence in the New Forest in $22.6 \%$. Lyme serology was performed in $81.7 \%$ of patients, of which $29 \%$ were positive. Antibiotics were prescribed for $73.1 \%$ of patients, oral steroids for $44 \%$ and aciclovir for $17.2 \%$.

Conclusion: Lyme disease is a significant cause of FNP in this endemic area of the UK, and there was a large degree of variability in management prior to national guideline publication. Areas with endemic Lyme disease should consider introducing local guidelines supporting routine investigation and management for FNP, including empiric treatment for Lyme disease in accordance with NICE guidelines to improve care and reduce variability. 


\section{Introduction}

Facial nerve palsy (FNP) is an uncommon presentation in paediatrics with a reported incidence of 25 per 10,000 per year in the UK (1). Due to its dramatic appearance, FNP is of a great concern to both caregivers and children. Whilst in adults most cases of unilateral facial weakness are idiopathic (2), in children there is usually an identifiable cause. Although acquired cases of FNP have historically mostly been attributed to otitis media, there is more recent evidence that in endemic areas, Lyme disease has become the most commonly identified paediatric cause $(3,4)$.

Many Lyme disease cases occur near forested areas (5). University Hospital Southampton NHS Foundation Trust is the major hospital providing secondary and tertiary paediatric care adjacent to the New Forest National park. The New Forest and surrounding region is home to many deer and other wildlife which can harbour Ixodes ricinus, a tick which is the primary vector for Borrelia burgdorferi(6). Up to $30 \%$ of Ixodes ricinus ticks are seropositive for Borrelia burgdorferi in some areas (7). As well as a high prevalence of ticks, the New Forest contains many towns and villages, and has a high number of human tourist visits making it a "UK hotspot" for cases of Lyme Disease(8).

Facial nerve palsy in combination with Lyme disease has been shown to cluster in the South of England especially in the New Forest and Exmoor areas(5). It is also commoner in younger age groups with the highest numbers occurring in children under 10 years of age (5).

The UK National Institute for Health and Care Excellent (NICE) is an executive body which publishes evidence based appraisals, and has recently produced guidelines for clinicians on the investigation and management of Lyme disease(9). We sought to capture how paediatricians locally were investigating and managing causes of FNP that appeared idiopathic at presentation, to establish the degree of variation in management, and to establish whether Lyme disease was being considered as a cause and being investigated and treated appropriately.

\section{Methods}

We conducted a retrospective review of the medical records including children under 18 years of age presenting to the emergency department (ED), or paediatric assessment unit (PAU) with a FNP between January $1^{\text {st }} 2010$ and December $31^{\text {st }} 2017$. Exclusion criteria were FNP with forehead sparing (upper motor neuron lesions), a known iatrogenic cause (e.g. post-operative, forceps delivery), a known neurological co-morbidity associated with FNP (e.g. malignancy, epilepsy, cerebral palsy), or a known traumatic mechanism directly affecting the facial nerve. We excluded patients for whom their FNP was not their presenting complaint (patients with pre-existing FNP) or patients with subsequent presentations with the same FNP. We collected data on demographics including age, gender and duration of symptoms prior to presentation. We collected data on clinical presentation including signs and symptoms such as recent travel to or residence in the New Forest; recent illness, defined by parent/caregiver reporting a period of the child either being generally unwell or affecting focal systems (which may be indicative of a prodrome); history of a tick bite or history of a rash. As the rash was reported by parents/caregivers we did not (and were unable to) discriminate between rashes diagnosed as erythema multiforme or other rashes. We collected further data on investigations including Lyme serology and imaging, and management including all medications. Both electronic and scanned paper records were reviewed (by RD, DO, DS and AM). 
Lyme serology was performed locally by enzyme-linked immunosorbent assay. After 2013, all patients who screened positive locally for IgM or IgG had samples forwarded to the Lyme national reference laboratory for England and Wales (the Public Health England Rare Imported Pathogens Laboratory, RIPL) for immunoblot testing. This is recommended as part of standard 2 tier serological investigation for Lyme disease due to its increased sensitivity and specificity over ELISA alone(10), which can be falsely negative in early disease, and may be positive due to cross reactivity.

As the study was performed as a service evaluation exercise, formal ethical approval was not required. Descriptive statistics were performed using RStudio (v1.1.456.).

\section{Results}

\section{Demographics}

Electronic hospital record systems returned 157 potential patients presenting with an FNP in the time period of interest. After exclusions (see above and figure 1), there were 93 patients included in the analysis. 44 were male (47.3\%) and 49 female (52.7\%). The median age at presentation was 9.3 years (IQR 4.6 - 12 years). Just over half of patients presented within 24 hours of onset of symptoms (48 of $92,51.6 \%$ ), with only a small number of patients presenting after 7 days ( 4 of $93,4.3 \%$ ).

\section{Presentation}

Elements of the history which consistent with a history of Lyme disease(9) were present in several patients, including recent travel or residence in the New Forest (21 of 93, 22.6\%), recent illness (31 of $93,33.3 \%)$, history of a tick bite $(13$ of $93,14 \%)$ and history of a rash $(5$ of $93,5.4 \%)$ In addition, a history of recent otitis media (within 2 weeks) was present in 12 of 93 (12.9\%).

\section{Investigations}

Investigations performed included Lyme serology for 76 of 93 (81.7\%) patients, which were positive in 22 of 76 (29\%) of those tested. Results of the 15 patients who had positive tests at RIPL are presented (see Table 1).

Neuroimaging was performed in 18 of 93 (19.4\%) children, with abnormalities present in 7 of 18 $(38.9 \%)$, including findings consistent with inflammation or infection of the facial nerve.

\section{Management}

There was a high degree of variability among treatments used. Antibiotics were prescribed for 68 of 93 (73.1\%), the majority of which were oral amoxicillin (54 of $68,79.4 \%)$. Course duration of antibiotics ranged from 1 day to 28 days, with a median of 14 days (IQR $12-14$ days). Oral prednisolone was prescribed for 41 of 93 (44\%) of patients and aciclovir was prescribed for 16 of 93 (17.2\%). Eye lubrication was prescribed for 73 of 93 (78.5\%)

\section{Discussion}

The demographics of our cohort were consistent with the literature, including a predominance in children under 10 years of age (11). Although many of the features of Lyme disease are non-specific, factors in the medical history which would increase suspicion for Lyme disease were present in a significant number of patients. The number of patients tested for Lyme serology (81.7\%) likely represents local knowledge regarding the increased incidence of Lyme disease in the region due to proximity to the New Forest. However, despite this nearly 1 in 5 patients were not tested. Even in the absence of other signs or symptoms of Lyme disease, an FNP could be the sole presenting sign (5). A high number of patients had positive Lyme serology in our cohort ( $29 \%$ of those tested), which is consistent with previous research from Lyme endemic areas finding positive Lyme serology in 30 - 
$34 \%$ of children with facial nerve palsy $(12,13)$. This prevalence is high enough that all children presenting with facial nerve palsy in endemic areas should have Lyme serology performed. This is particularly important as it has been demonstrated that clinical features alone perform poorly at being able to determine the likelihood of Lyme aetiology(14), and outcomes following treatment of Lyme disease are excellent, recently confirmed by large adult population studies in Europe of Lyme neuroborreliosis(15).

We found significant variation in medical management, with some children appearing to receive no treatment, and others receiving antibiotics, steroids and antivirals. Other studies have also demonstrated a significant amount of variation in the management of Lyme FNP in other institutions(16). Although antibiotics were prescribed for nearly three quarters of patients, considering the high number of patients with positive Lyme serology, our new regional guideline for FNP (17) recommends starting all children with FNP on empirical therapy for Lyme disease whilst awaiting serology results. This is based on the NICE Lyme treatment recommendations(9). Given the high percentage of cases due to Lyme disease, our guidelines also recommend sending all samples for western blot, regardless of whether the initial ELISA was negative, due to the possibility of false negatives from ELISA alone. Patients are continued on antibiotics unless they test negative via western blot.

Given that none of these patients had features of a definitive cause of their FNP at presentation, the degree of variability in management appears unjustified and inconsistency has potential to cause confusion amongst patients and caregivers. This variability may reflect the paucity of evidence and controversy surrounding the efficacy of steroids and antivirals, and that the prognosis of FNP is generally good in children (18-21).

The main strength of our study include that it looked at a wide time period, and our is that our screening search criteria was broad meaning few, if any, cases of FNP would have been missed. Limitations of our study include its retrospective nature, and due to variations in clinical records some information was unobtainable for some patients. This also impaired our ability to determine other features of interest, including outcomes. It has however been established that outcomes of childhood facial nerve palsy (such as time to resolution) are excellent in general, so are less important in this regard (21). Although the duration of symptoms prior to onset of symptoms was known, no studies of Lyme presenting to primary or secondary care are able to accurately determine the timing according to exposure to tick bite, as even if a tick bite had been noticed it is not possible to say for certain whether the infection was transmitted by that tick or another, on that or a different occasion. It is therefore possible that testing may have occurred early in the illness, increasing the possibility of false negative serology results. A further limitation is that the number of patients in our sample is relatively small, however it is representative of presentations over a wide time period from a Lyme endemic area which increases its generalisability.

\section{Conclusion}

In areas endemic with Lyme disease, Lyme disease should be considered as the likely cause of facial nerve palsy in children until proven otherwise. All children presenting with FNP to health care providers in these areas should have Lyme serology tested and empirical treatment for Lyme initiated pending the results of tests. 


\section{References}

1. Rowlands S, Hooper R, Hughes R, Burney P. The epidemiology and treatment of Bell's palsy in the UK. Eur J Neurol. 2002;9(1):63-7.

2. Peitersen E. Bell's palsy: the spontaneous course of 2,500 peripheral facial nerve palsies of different etiologies. Acta Otolaryngol Suppl. 2002(549):4-30.

3. Cook SP, Macartney KK, Rose CD, Hunt PG, Eppes SC, Reilly JS. Lyme disease and seventh nerve paralysis in children. Am J Otolaryngol. 1997;18(5):320-3.

4. Tveitnes D, Oymar K, Natas O. Acute facial nerve palsy in children: how often is it lyme borreliosis? Scand J Infect Dis. 2007;39(5):425-31.

5. Cooper L, Branagan-Harris M, Tuson R, Nduka C. Lyme disease and Bell's palsy: an epidemiological study of diagnosis and risk in England. Br J Gen Pract. 2017;67(658):e329-e35.

6. Jameson L, Medlock JM. Tick surveillance in Great Britain. Vector Borne Zoonotic Dis. 2011;11(4):403-12.

7. Medlock JM, Hansford KM, Vaux AGC, Cull B, Gillingham E, Leach S. Assessment of the Public Health Threats Posed by Vector-Borne Disease in the United Kingdom (UK). Int J Environ Res Public Health. 2018;15(10).

8. Dobson AD, Taylor JL, Randolph SE. Tick (Ixodes ricinus) abundance and seasonality at recreational sites in the UK: hazards in relation to fine-scale habitat types revealed by complementary sampling methods. Ticks Tick Borne Dis. 2011;2(2):67-74.

9. (NICE) NIfHaCE. Lyme Disease. NICE; 2018.

10. Centers for Disease C, Prevention. Recommendations for test performance and interpretation from the Second National Conference on Serologic Diagnosis of Lyme Disease. MMWR Morb Mortal Wkly Rep. 1995;44(31):590-1.

11. Evans AK, Licameli G, Brietzke S, Whittemore K, Kenna M. Pediatric facial nerve paralysis: patients, management and outcomes. Int J Pediatr Otorhinolaryngol. 2005;69(11):1521-8.

12. Nigrovic LE, Thompson AD, Fine AM, Kimia A. Clinical predictors of Lyme disease among children with a peripheral facial palsy at an emergency department in a Lyme disease-endemic area. Pediatrics. 2008;122(5):e1080-5.

13. Kanerva M, Nissinen J, Moilanen K, Maki M, Lahdenne P, Pitkaranta A. Microbiologic findings in acute facial palsy in children. Otol Neurotol. 2013;34(7):e82-7.

14. Nigrovic LE, Bennett JE, Balamuth F, Levas MN, Chenard RL, Maulden AB, et al. Accuracy of Clinician Suspicion of Lyme Disease in the Emergency Department. Pediatrics. 2017;140(6).

15. Obel N, Dessau RB, Krogfelt KA, Bodilsen J, Andersen NS, Moller JK, et al. Long term survival, health, social functioning, and education in patients with European Lyme neuroborreliosis: nationwide population based cohort study. BMJ. 2018;361:k1998.

16. Paydar-Darian N, Kimia AA, Lantos PM, Fine AM, Gordon CD, Gordon CR, et al. Diagnostic Lumbar Puncture Among Children With Facial Palsy in a Lyme Disease Endemic Area. J Pediatric Infect Dis Soc. 2017;6(2):205-8.

17. Bhangu N. Pier NetworkFacial Nerve Palsy Guideline 2016 [December 2018]. Available from: https://www.piernetwork.org/uploads/4/7/8/1/47810883/facial-nerve-palsy-guideline.pdf.

18. Gagyor I, Madhok VB, Daly F, Somasundara D, Sullivan M, Gammie F, et al. Antiviral treatment for Bell's palsy (idiopathic facial paralysis). Cochrane Database Syst Rev. 2015(11):Cd001869.

19. Zandian A, Osiro S, Hudson R, Ali IM, Matusz P, Tubbs SR, et al. The neurologist's dilemma: a comprehensive clinical review of Bell's palsy, with emphasis on current management trends. Med Sci Monit. 2014;20:83-90.

20. Ashtekar CS, Joishy M, Joshi R. Best evidence topic report. Do we need to give steroids in children with Bell's palsy? Emerg Med J. 2005;22(7):505-7.

21. McNamara R, Doyle J, Mc Kay M, Keenan P, Babl FE. Medium term outcome in Bell's palsy in children. Emerg Med J. 2013;30(6):444-6. 
Table 1. Reference laboratory results for 15 children with positive $\mathrm{C} 6$ tests indicating Lyme Disease.

\begin{tabular}{|c|c|c|c|}
\hline Gender & Age (yrs) & C6 (EIA index)* & Immunoblot (positive bands) \\
\hline Male & 7.4 & 8.1 & IgG OspC VISE IgM OspC VISE \\
\hline Female & 10.8 & 5.3 & IgG VISE IgM p41 \\
\hline Male & 3 & 6.1 & IgG OspC VISE IgM OspC \\
\hline Female & 9.2 & 7.4 & IgG OspC VISE IgM p41 OspC \\
\hline Female & 3.9 & 8.4 & IgG VISE IgM OspC \\
\hline Male & 9.1 & 5.4 & IgG OspC \\
\hline Male & 12 & 7.5 & IgG VISE \\
\hline Female & 10.1 & 8.5 & IgG VISE \\
\hline Male & 4.6 & 7.2 & IgG p21VISE IgM OspC \\
\hline Female & 4.9 & 8.2 & IgG p30 VISE IgM p41 \\
\hline Male & 6.4 & 8.8 & IgG p39 OspC VISE IgM OspC VISE \\
\hline Female & 2.9 & 8.2 & IgG VISE \\
\hline Male & 12.1 & 8.5 & IgG inhibitory IgM p41 OspC \\
\hline Male & 6.7 & 7.9 & IgG inhibitory IgM Neg \\
\hline Male & 6.8 & 5.2 & IgG VISE IgM p41 OspC Oscp17 \\
\hline
\end{tabular}

${ }^{*}$ C6 Positive $>1.1$ 


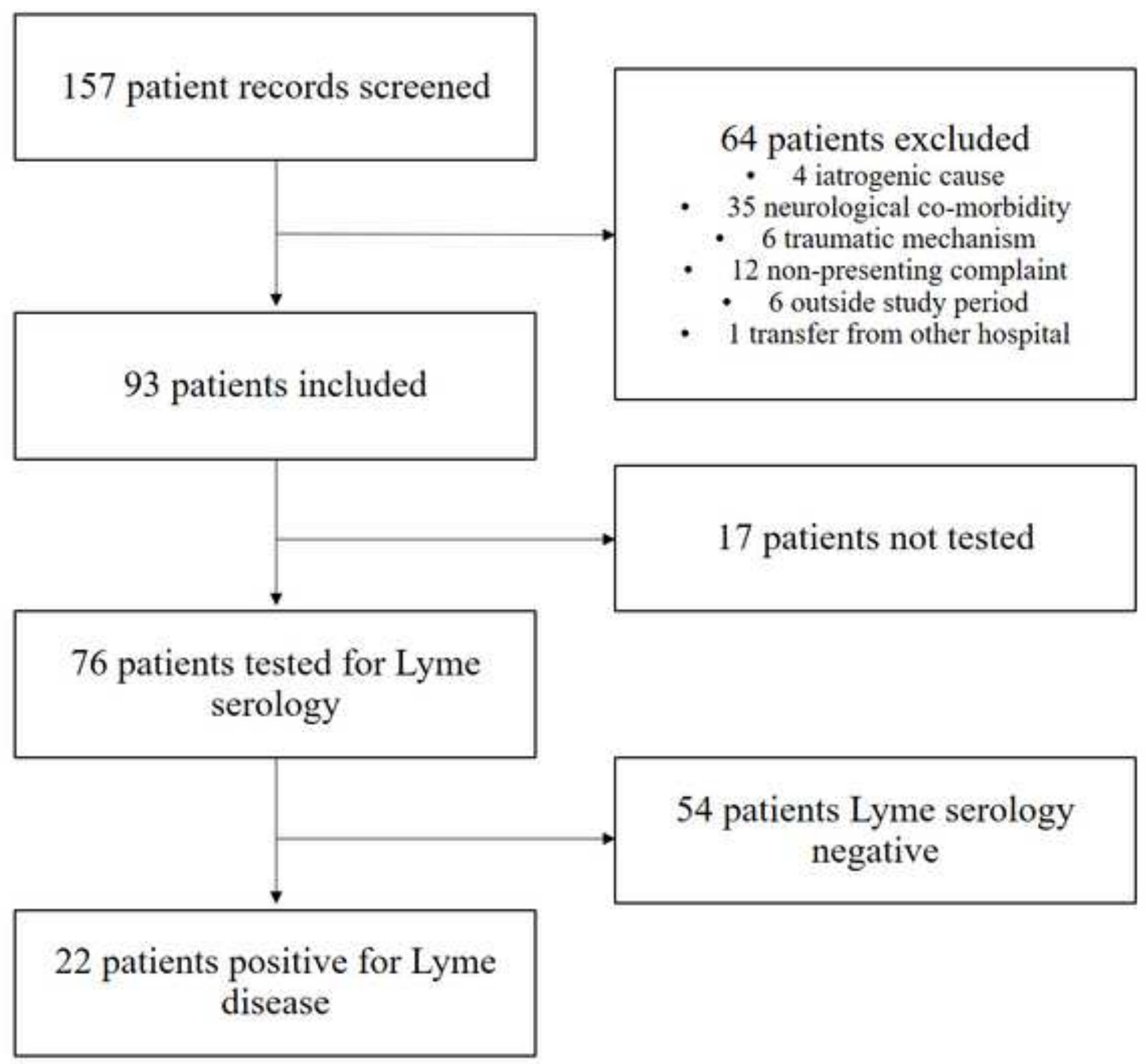

